See discussions, stats, and author profiles for this publication at: https://www.researchgate.net/publication/276415282

\title{
Service compris... à certaines conditions
}

Article in L Expansion Management Review · January 2014

DOI: $10.3917 /$ emr.154.0043

4 authors, including:

Olivier Furrer

Université de Fribourg

165 PUBLICATIONS 3,219 CITATIONS

SEE PROFILE

(2) Brian Tjemkes

77 PUBLICATIONS 185 CITATIONS

SEE PROFILE

Meindert Flikkema

Vrije Universiteit Amsterdam

10 PUBLICATIONS 219 CITATIONS

SEE PROFILE

Some of the authors of this publication are also working on these related projects:

Strategic Groups View project

Project Product Services Strategies / Services autour des produits View project 


\title{
Service compris... à certaines conditions
}

\author{
DE PLUS EN PLUS D'ENTREPRISES INDUSTRIELLES AJOUTENT DES SERVICES À LEUR OFFRE POUR SE \\ DIFFÉRENCIER. LES RÉSULTATS D'UNE ÉTUDE EMPIRIQUE SUR LEURS CHOIX ET LEUR PERFORMANCE.
}

\section{$>$ Olivier Furrer, Brian Tjemkes, Meindert Flikkema et Goran Pisic}

$\mathbf{F}$ ournir des services autour de leurs produits est devenu une stratégie de plus en plus prisée par les entreprises manufacturant ou vendant des biens tangibles. On observe cependant une controverse autour de ce type de stratégie parce que la relation entre offre de services et performance est ambiguë. Une stratégie de services autour des produits est supposée permettre d'obtenir un avantage concurrentiel, mais de nombreuses entreprises ont constaté que ces services engendraient des coûts supplémentaires non compensés par une augmentation des ventes. Dans cet article, nous présentons les résultats d'une étude empirique qui démontre que les firmes qui veulent fournir des services autour de leurs produits doivent prendre en compte le contexte environnemental et les objectifs stratégiques de l'entreprise. Ce n'est qu'à cette condition qu'une telle politique peut être performante.

Bobst, une entreprise qui reconditionne ses machines pour en allonger la durée de vie. Bobst est une entreprise suisse, leader mondial des machines pour fabricants d'emballages. Avec son service Boost my BOBST, elle offre à ses clients ${ }^{(1)}$ de développer le potentiel et les fonctionnalités de leurs machines. Grâce à ce service, ceux-ci peuvent améliorer la qualité, booster la productivité, augmenter la disponibilité,

\section{Les points forts}

Si une stratégie de services autour des produits est supposée permettre d'obtenir un avantage concurrentiel, elle engendre aussi des coûts pas toujours compensés par une augmentation des ventes ou de la rentabilité.

Les entreprises qui s'engagent dans cette voie doivent choisir une orientation qui corresponde aux caractéristiques de leurs produits et de leur contexte.

Cinq stratégies de services peuvent se montrer efficaces, mais à certaines conditions et pour des critères déterminés de performance en termes de rentabilité, de ventes ou de satisfaction des clients.

optimiser l'ergonomie ou renforcer la sécurité de leurs machines, même vieilles de quinze ou vingt ans.

Nike, une entreprise qui permet à ses clients de personnaliser leurs chaussures et équipements. A travers son service NIKEiD ${ }^{(2)}$, Nike propose à ses clients de devenir codesigners des produits qu'ils achètent. Grâce à ce service disponible en ligne ou dans des studios installés en $\gg$

Olivier Furrer (olivier.furrer@unifr.ch) est professeur de marketing à l'université de Fribourg en Suisse, Brian Tjemkes (b.v.tjemkes@vu.nl) est professeur associé en management stratégique à l'université libre d'Amsterdam aux Pays-Bas, Meindert Flikkema (m.j.flikkema@vu.nl) est professeur associé en management de l'innovation dans les services à l'université libre d'Amsterdam. Goran Pisic (g.pisic@vu.nl) est étudiant en master en stratégie et organisation à l'université libre d'Amsterdam. 
$\gg$ magasins, les consommateurs peuvent adapter le style de leurs chaussures et équipements, en choisir les couleurs et y faire inscrire leur nom.

Michelin, une entreprise qui vend des kilomètres. Michelin Fleet Solution ${ }^{(3)}$ propose aux transporteurs non plus de leur vendre des pneus mais de les leur louer. Michelin facture ses services en fonction des kilomètres parcourus. Le fabricant met ainsi son expertise à la disposition de ses clients, pour qui l'entretien des pneus tout au long de leur cycle de vie n'est pas le métier, même si cet entretien a un impact important sur leur performance (un pneu sous-gonflé de $10 \%$ augmente la consommation du véhicule de $1 \%$ et s'use plus rapidement).

Heineken, une entreprise qui achète et reloue des bars et des pubs pour assurer la distribution de ses bières. Depuis 2012 en Angleterre, Heineken, à travers sa filiale Star Pubs \& Bar ${ }^{(4)}$, loue des pubs et des bars à des patrons indépendants. En rachetant ces espaces dont la localisation est stratégique pour la marque et en les mettant à la disposition de tenanciers qui deviennent ses partenaires exclusifs, Heineken s'assure un plus grand pouvoir sur l'ensemble de la chaîne de distribution et un meilleur contrôle sur la distribution, les prix et la publicité de ses produits.

Harley-Davidson, une entreprise qui offre à ses clients de vivre l'expérience Harley et pas seulement d'utiliser la moto pour se déplacer. Grâce à ses clubs ${ }^{(5)}$ et aux événements $H-D^{\circledR}{ }^{(6)}$, la marque offre à ses fans une expérience faite de liberté et d'aventure.

\section{Le résultat d'une cascade de transformations}

Ces différents exemples de stratégies de services autour de produits sont le résultat d'une cascade de transformations majeures qui ont révolutionné le marketing des services au cours des trente dernières années : (1) la «servitization» des entreprises industrielles ; (2) le passage d'un marketing transactionnel à un marketing relationnel; (3) le développement d'une nouvelle logique marketing dominée par les services; et (4) l'avènement $d$ 'un marketing des services qui se concentre sur les expériences vécues par les consommateurs.

Comme exposé ailleurs ${ }^{(7)}$, l'intensification de la concurrence par les prix due à une saturation de certains marchés et à la recherche d'économies d'échelle, le développement et la baisse des coûts des nouvelles technologies de l'information qui permettent d'offrir des services à distance de plus en plus facilement, et une tendance à l'uniformisation des offres de produits manufacturés poussée par la mondialisation ont contribué au développement d'un phénomène que Vandermerwe et Rada ${ }^{(8)}$ ont appelé la «servitization » des entreprises industrielles et qui consiste pour celles-ci à créer des services autour de leurs pro-

>(1) http://www.bobst.com/frfr/services/boost-my-bobst/\#. U5GCHBbA3Zo

$>$ (2) http://www.nike.com/fr/fr_fr/c/nikeid

(3) http://www.michelintransport.com/ple/front/affich. jsp?codeRubrique $=20051018154228 \&$. Voir aussi : http://curiosites-strat.blogspot.ch/2008/12/michelin-fleetsolution-le-pneu-au.html

$>$ (4) http://www.starpubs.co.uk/about-us

$>$ (5) http://www.fhdce.eu/index2.html

>(6) http://www.harley-davidson.com/content/h-d/fr_FR/ home/events.html

>(7) O. Furrer, "Le rôle stratégique des "services autour des produits" ", Revue française de gestion, $n^{\circ} 113,1997$. $>$ (8) S. Vandermerwe et J. Rada, "Servitization of Business : Adding Value by Adding Services ", European Management Journal, vol. 6, $n^{\circ} 4,1988$. Voir aussi : R. Oliva et R. Kallenberg, "Managing the Transition from Products to Services ", International Journal of Service Industry Management, vol. 14, $n^{\circ} 2,2003$; F. Jacob et W. Ulaga, "The Transition from Product to Service in Business Markets : An Agenda for Academic Inquiry ", Industrial Marketing Management, vol. 37, $\mathrm{n}^{\circ} 3,2008$; et T. S. Bain, H. W. Lightfoot, O. Benedettini et J. M. Kay, "The Servitization of Manufacturing : A Review of Literature and Reflection on Future Challenges", Journal of Manufacturing Technology Management, vol. 20, n 5, 2009. 
duits afin de se démarquer de leurs concurrents et de différencier leurs offres. C'est le cas de Michelin.

Ce phénomène s'est accompagné d'un changement dans l'approche marketing d'un nombre croissant d'entreprises, comme Bobst et Heineken, par le passage d'un marketing transactionnel, focalisé sur la vente et l'acquisition de nouveaux clients, à un marketing relationnel, dont l'objectif principal est de fidéliser les clients en entretenant et développant des relations partenariales à long terme, mutuellement profitables. Les services jouent un rôle particulièrement important dans le

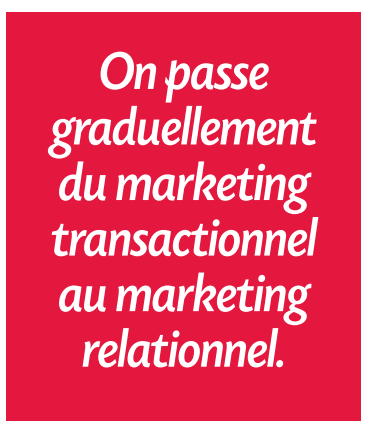

sur les expériences vécues par leurs consommateurs dans leurs relations avec elles ${ }^{(11)}$. Afin d'améliorer cette expérience, ces entreprises gèrent l'ensemble des points de contact par lesquels leurs clients interagissent avec elles, que ce soit en personne ou à travers des canaux digitaux. Dans le cadre de ce marketing dit expérientiel, les effets physiologiques, psychologiques, émotionnels et culturels des relations entre une entreprise et ses clients sont pris en compte ${ }^{(12)}$, et les services jouent là un rôle primordial.

Ces différentes révolutions ont entraîné des changements dans les stratégies des entremaintien de ces relations parce qu'ils sont plus à même que les produits de permettre une interaction étroite entre les entreprises et leurs clients ${ }^{(9)}$.

Ces changements dans la pratique des entreprises et leurs modes de concurrence ont amené certains chercheurs à réfléchir sur la nature même du marketing et à proposer une nouvelle logique dominée par les services ${ }^{(10)}$. L'idée fondamentale de cette nouvelle logique, c'est que les entreprises et les marchés sont fondamentalement concernés par l'échange de services plutôt que de biens. Autrement dit, des services sont échangés contre des services et toutes les entreprises sont en fait des entreprises de services. Par conséquent, la pensée et la pratique marketing doivent reposer sur des théories, principes et logiques de service. Dans cette nouvelle logique marketing, tout service est en fait coproduit, c'est-à-dire que le client participe à sa conception et à sa réalisation, ne serait-ce que par sa simple présence (comme dans l'exemple de Nike).

Au-delà de prix bas et de produits innovants, au-delà de relations de services partenariales et interactives, des entreprises, comme Harley-Davidson, se sont rendu compte de l'importance de se concentrer prises industrielles. A la suite de leaders comme IBM pour qui les services représentent déjà plus de $50 \%$ de son chiffre d'affaires ${ }^{(13)}$, un grand nombre d'entre elles ont mis en place des stratégies de services autour de leurs produits.

Ces services sont fournis complémentairement à un produit en vue d'en faciliter le choix et l'acquisition, d'en optimiser l'utilisation et d'en augmenter la valeur pour les clients ${ }^{(14)}$. Ils procurent des avantages aux entreprises qui les offrent en contribuant directement et indirectement

\footnotetext{
> (9) Voir, par exemple, L. L. Berry, "Relationship Marketing of Service - Growing Interest, Emerging Perspectives ", Journal of the Academy of Marketing Science, vol. 23, n 4, 1995.

$>$ (10) S. L. Vargo et R. F. Lusch, "Evolving to a New Dominant Logic for Marketing ", Journal of Marketing, vol. 68, $n^{\circ}$ 1, 2004 ; "From Goods to Service(s) : Divergences and Convergences of Logics ", Industrial Marketing Management, vol. 37, $n^{\circ} 3,2008$.

> (11) B. J. Pine et J. H. Gilmore, The Experience Economy: Work is Theatre \& Every Business a Stage, Harvard Business Press, 1999.

> (12) P. C. Verhoef, K. N. Lemon, A. Parasuraman, A. Roggeveen, M. Tsiros et L. A. Schlesinger, " Customer Experience Creation : Determinants, Dynamics and Management Strategies ", Journal of Retailing, vol. 85, $n^{\circ} 1,2009$. > (13) H. Gebauer, B. Edvardsson, A. Gustafsson, et L. Witell, " Match or Mismatch : Strategy-Structure Configurations in the Service Business of Manufacturing Companies ", Journal of Service Research, vol. 13, n², 2010. $>$ (14) O. Furrer, 1997, op. cit. ; et O. Furrer, "Stratégies de services autour des produits ", in Ph. Callot (dir.), Marketing des services, Vuibert, 2007.
} 
$\gg$ aux profits. Directement parce qu'ils sont souvent très rentables et indirectement parce qu'ils favorisent la vente des produits qu'ils entourent. Les services autour des produits sont souvent une condition pour la vente des produits tangibles et un catalyseur de la relation entre l'entreprise et ses clients. De plus, certains services permettent de desserrer la pression sur les prix en différenciant l'offre de celle des concurrents, de dresser des barrières à l'entrée grâce aux investissements qu'ils nécessitent et d'établir une certaine dépendance des clients envers l'entreprise à cause des coûts qu'entraîne tout changement de fournisseur.

De nombreux articles sur les stratégies de services autour des produits ont été publiés ces vingt dernières années ${ }^{(15)}$. Mais cette littérature n'en est encore qu'à un stade embryonnaire. Certains auteurs se bornent à définir le concept de services autour des produits, d'autres à en dresser une typologie, d'autres encore ont identifié les différentes mises en place par les entreprises. Même si l'importance stratégique de ces services pour les entreprises fabriquant ou commercialisant des produits tangibles n'est plus remise en question ${ }^{(16)}$, très peu d'attention a encore été accordée aux critères de choix et aux conditions d'application de ces stratégies. Une question fondamentale qui pour l'instant reste encore en suspens est la suivante: quelle stratégie de service autour des produits dois-je mettre en place et dans quelles conditions?

\section{Les lacunes des études existantes}

La grande majorité des études académiques mettent en avant les avantages concurrentiels et financiers que les entreprises peuvent retirer de la mise en place d'une stratégie basée sur les services autour des produits, mais plusieurs études empiriques pointent l'existence d'un paradoxe ${ }^{(17)}$. Si une telle stratégie est supposée permettre d'obtenir un avantage concurrentiel, de nombreuses entreprises se sont aperçues que ces services engendraient des coûts supplémentaires non compensés par une augmentation des ventes. En particulier, les études empiriques de Gebauer, Fleisch et Friedli et de Neely ont montré que l'ajout de services autour des produits entraîne souvent une hausse des coûts et une diminution du taux de rentabilité.

Cette divergence entre arguments théoriques et résultats empiriques est problématique pour les managers et vexante pour les chercheurs. Plusieurs lacunes dans les études existantes peuvent l'expliquer.

Des objectifs stratégiques mal repérés. $\mathrm{La}$ première limite des études existantes provient du fait que nombre d'entre elles ne définissent pas de façon très claire les objectifs dévolus à ces services ou mélangent des objectifs financiers, stratégiques et marketing ${ }^{(18)}$. Deux objectifs bien distincts peuvent être attribués aux services : attirer de nouveaux clients et fidéliser les

$>$ (15) Pour une revue de la littérature récente, voir O. Furrer, "A Customer Relationship Typology of Product Services Strategies ", in F. Gallouy et F. Djellal (dir.), The Handbook of Innovation and Services : A Multi-Disciplinary Perspective, Edward Elgar Publishing, 2010.

$>$ (16) Notamment depuis les travaux d'O. Furrer ( $\mathrm{d}^{\prime} \mathrm{The}$ Strategic Role of Product Services : Attract New Customers vs. Retain Existing Ones ", $11^{\text {th }}$ Annual AMA Frontiers in Services Conference, 27-29 juin 2002, Maastricht) qui montrent que les entreprises qui mettent en place une stratégie de services autour des produits se différencient plus facilement de leurs concurrents et bénéficient d'une plus grande fidélité de leur clientèle.

$>(17)$ H. Gebauer, E. Fleisch et T. Friedli, "Overcoming the Service Paradox in Manufacturing Companies ", European Management Journal, vol. 23, $\mathrm{n}^{\circ} 1,2005$; ou encore A. Neely, "Exploring the financial Consequences of the Servitization of Manufacturing ", Operations Management Research, vol. 1, n², 2008.

$>$ (18) Voir R. Oliva et R. Kallenberg, 2003, op. cit. ; et H. Gebauer et T. Friedli, "Behavioral Implications of the Transition Process from Products to Services ", Journal of Business \& Industrial Marketing, vol. 20, n², 2005. 
clients existants. Si ces objectifs ne sont pas toujours incompatibles, ils requièrent le plus souvent des services bien différents. Evaluer la performance d'une stratégie de services autour des produits sans tenir compte des objectifs recherchés par les entreprises n'a donc que peu de sens et a de fortes chances d'aboutir à des résultats inconsistants.

D Une variété d'indicateurs de performance. Le manque de clarté sur les objectifs a pour corollaire l'utilisation d'une grande variété d'indicateurs pour en évaluer la performance. Certaines études ont employé des indicateurs de performance financière comme le taux de rentabilité(19), d'autres ont mesuré la performance stratégique en termes d'avantage concurrentiel ou de différentiation ${ }^{(20)}$, d'autres encore ont adopté des indicateurs de performance marketing ${ }^{(21)}$ comme l'image de marque ou la satisfaction des clients. Il est cependant important d'aligner les indicateurs de performance sur les objectifs dévolus aux services. Si ces services ont pour objectif d'attirer de nouveaux clients, alors des indicateurs de performance comme la croissance du chiffre d'affaires sont bien adaptés. Mais si leur objectif principal est d'améliorer la fidélité des clients, alors il vaut mieux utiliser des indicateurs marketing.

Des études a-contextuelles. Le contexte des entreprises qui fournissent des services autour de leurs produits a souvent été négligé dans les études portant sur les stratégies de services. Un des critères les plus importants pour la performance d'une stratégie de services autour des produits concerne les caractéristiques du produit lui-même. En effet, un produit technologiquement complexe va nécessiter d'autres services qu'un produit plus simple. De même, offrir des services aux particuliers ou aux entreprises est quelque chose de très différent. Là où les entreprises clientes sont prêtes à payer pour des services qui sont hors de leur champ de compétences, les particuliers préfèrent souvent recevoir les services gratuitement même si leur prix est inclus dans le prix du bien qu'ils achètent. Ne pas distinguer entre stratégie de services B2B et B2C a de fortes chances de créer des problèmes de comparaison.

D Principalement des études de cas. Une autre faiblesse importante des études existantes tient au fait qu'elles sont pour la plupart basées sur un nombre limité d'études de cas (22). Par exemple, Davies, Brady et Hobday (23) fondent leurs recommandations sur cinq cas d'entreprises leaders et Oliva et Kallenberg (24) sur onze. Pareillement, Gebauer et Friedli ${ }^{(25)}$ ont étudié cinq entreprises ayant réussi la mise en place d'une stratégie de services et six entreprises ayant eu des difficultés avec leurs services. Le manque de représentativité de ces travaux requiert des études quantitatives sur des échantillons plus importants.

En résumé, les études existantes souffrent de quatre limites principales : un manque de clarté dans les objectifs dévolus aux services autour des produits, des critères de performance qui ne sont pas toujours adaptés aux objectifs poursuivis, une non-prise en compte du contexte environnemental, et des résultats basés sur des échantillons très restreints. Afin de pallier

>(19) V. Malleret, "Value Creation through Service Offer", European Management Journal, vol. 24, $\mathrm{n}^{\circ}$ 1, 2006.

$>(20)$ R. T. Frambach, I. Wels-Lips, et A. Guendlach, "Proactive Product Service Strategies : An Application in the European Health Market ", Industrial Marketing Management, vol. 26, n4, 1997.

>(21) S. Vandermerwe et J. Rada, 1988, op. cit.

$>(22)$ T. S. Bain, H. W. Lightfoot, O. Benedettini et J. M. Kay, 2009, op. cit.

>(23) A. Davies, T. Brady et M. Hobday, "Charting a Path toward Integrated Solutions ", MIT Sloan Management Review, vol. 47, $\mathrm{n}^{\circ} 3,2006$.

$>$ (24) R. Oliva et R. Kallenberg, 2003, op. cit.

>(25) H. Gebauer et T. Friedli, 2005, op. cit. 
$\mathbf{U}$ ne typologie de stratégies de services autour des produits a d'abord été créée sur la base d'une revue de la littérature et d'entretiens avec quelques managers, qui ont aussi permis d'identifier les objectifs dévolus à ces services et le contexte environnemental et industriel de ces stratégies. Dans un second temps, pour chacune de ces stratégies, des échelles de Likert ont été développées, ainsi que des échelles de mesure pour les différents objectifs et conditions d'application de ces stratégies. Les échelles et le questionnaire ont ensuite été validés avec un panel d'experts académiques.

Le questionnaire élaboré a été administré dans 82 entreprises. L'échantillon représentatif a été constitué à partir des membres d'une association professionnelle : $67 \%$ de ces entreprises ont plus de 50 employés, $60 \%$ ont une clientèle majoritairement industrielle (B2B) et $40 \%$ ont une clientèle majoritairement constituée de consommateurs (B2C) ; près de $80 \%$ d'entre elles retirent plus de $10 \%$ de leurs revenus des services.

Dans chaque entreprise, un manager responsable pour les services autour des produits a évalué l'offre de services de son entreprise, son contexte environnemental et sa performance. Après l'évaluation de la fiabilité et de la validité des échelles de mesure, l'analyse statistique d'un système d'équations structurelles a permis d'établir des relations entre la mise en place d'une des cinq stratégies et les conditions dans lesquelles elles étaient les plus performantes. ces faiblesses, nous avons conduit une étude empirique sur un échantillon représentatif d'entreprises offrant des services autour de leurs produits. L'objectif de cette étude était d'identifier différents types de stratégie de services autour des produits et leurs visées, puis d'identifier leurs critères de performance et leurs conditions d'application. C'est-à-dire dans quelles conditions telle ou telle stratégie est performante pour atteindre tel ou tel objectif.

\section{Cinq stratégies à la loupe}

Différentes typologies de stratégie de services ont été proposées dans la littérature ${ }^{(26)}$. La confrontation de ces typologies théoriques avec la pratique des entreprises lors d'entretiens avec des managers a permis d'identifier cinq manières d'utiliser les services autour des produits.

- Des services pour optimiser l'utilisation des produits. Cette stratégie consiste à offrir en option des services autour de produits relativement standard dans le but d'en améliorer les fonctionnalités ou d'en augmenter la durée de vie. C'est la stratégie de Bobst. L'objectif de cette stratégie est de rendre plus facile l'utilisation du produit par le client et cela tout au long de son cycle de vie. Il s'agit donc de fournir des services de formation à l'utilisation du produit, des services de maintenance et de réparation, ou même des services de recyclage du produit en fin de vie. Cette stratégie permet aux entreprises de différencier leur offre et de maintenir une relation à long terme avec leurs clients.

Des services pour personnaliser les produits. La seconde stratégie consiste à offrir aux consommateurs des services leur permettent de personnaliser le produit qu'ils achètent selon leurs besoins, désirs ou préférences. Cette stratégie peut se décliner de deux manières différentes en fonction du mode de personnalisation. Certaines entreprises, par

(26) Voir, par exemple, R. Oliva et R. Kallenberg, 2003, op. cit. ; A. Neely, 2008, op. cit. ; et O. Furrer, 2010, op. cit. 
exemple Nike, ont mis en place un système de personnalisation de masse qui donne aux clients la possibilité de choisir parmi des options prédéterminées. D'autres firmes proposent un système de personnalisation intensive sur la base d'un dialogue entre elles et leurs clients. Dans ce cas, elles cherchent à améliorer la satisfaction des clients en les faisant participer à la conception des produits. Cette stratégie vise à attirer de nouveaux clients qui ne sont pas satisfaits des offres standard des concurrents ou qui ont des besoins particuliers.

D Des services qui proposent des solutions plutôt que des produits. Cette stratégie permet aux clients de minimiser leur risque d'achat et d'utilisation d'un produit et de se concentrer sur leur métier de base.

Grâce à des prestations de services proposant des solutions aux préoccupations de leurs clients, les entreprises fournissent des résultats plutôt que des produits; de ce fait, elles tirent parti de la nouvelle logique marketing dominée par les services. Les services de Michelin appartiennent à cette catégorie. Cette stratégie donne à l'entreprise le moyen de développer des relations plus étroites avec ses clients et ainsi de les fidéliser. Elle présente de plus l'avantage de lui procurer des revenus réguliers, ce qui s'avère particulièrement utile dans un environnement caractérisé par des ventes de produits cycliques.

- Des services pour assurer la disponibilité des produits. L'objectif de cette stratégie est de supprimer les barrières à l'achat du produit tangible, en rapprochant ce produit des consommateurs et en en facilitant l'accès. Ces services ne sont pas nécessairement dirigés vers le consommateur final du produit mais peuvent être fournis tout au long de la chaîne de distribution afin de la rendre plus efficace. La stratégie de Heineken en est un exemple. Dans ce cadre, des services de conseil peuvent également être fournis au client final pour l'inciter à l'achat. L'objectif principal de cette stratégie est plus d'attirer de nouveaux clients que de les fidéliser.

Des services qui offrent une expérience de consommation globale. Cette stratégie cherche à développer une relation sociale avec les consommateurs en créant une expérience globale afin d'enrichir la relation qui se tisse entre le client et la marque, et ceci à plusieurs niveaux : rationnel, émotionnel, sensoriel, physique et spirituel ${ }^{(27)}$. Les clubs et événements Harley-Davidson font partie de cette stratégie. L'objectif de cette stratégie est essentiellement de fidéliser la clientèle plutôt que de chercher à attirer de nouveaux consommateurs. Le bouche-à-oreille des clients satisfaits de leur expérience permettra indirectement d'attirer de nouveaux clients.

\section{Les résultats d'une étude empirique}

Cette recherche a des implications très pratiques pour les entreprises qui souhaitent mettre en place une stratégie de services autour des produits. Les résultats empiriques montrent que les cinq stratégies décrites ci-dessus peuvent avoir un effet positif sur la performance de l'entreprise mais uniquement sous certaines conditions et selon des critères de performance déterminés.

Dans le premier cas, où il s'agit d'optimiser l'utilisation des produits, l'offre de services vise à attirer de nouveaux clients. Elle a un effet positif sur le chiffre d'affaires, mais seulement si les produits tangibles qu'ils entourent sont relativement standard. Elle permet de développer une

> (27) P. C. Verhoef, K. N. Lemon, A. Parasuraman, A. Roggeveen, M. Tsiros et L. A. Schlesinger, 2009, op. cit. 
$\gg$ relation financière avec les clients en leur offrant des services supplémentaires gratuits ou à un prix modique. Cette stratégie est en revanche peu efficace pour fidéliser les clients actuels car ceux qu'elle intéresse sont sensibles au prix et seront tentés de changer de fournisseur s'ils trouvent une offre plus attractive.

Dans le deuxième cas, l'offre de service permet, par la personnalisation des produits, d'attirer de nouveaux clients qui ne sont pas satisfaits des produits standard et par conséquent d'améliorer le chiffre d'affaires de l'entreprise. Cette stratégie est productive lorsque le marché est fragmenté et que les préférences des consommateurs changent rapidement. Par exemple, lorsqu'on a des effets de mode. Il est alors peu efficace d'offrir des produits standard. En revanche, permettre aux clients de participer au développement du design de leur produit en le personnalisant est particulièrement bénéfique.

Troisième cas de figure, offrir des solutions plutôt que des produits permet d'améliorer la rentabilité de l'entreprise en fidélisant la clientèle dont on augmente le panier moyen, plutôt que d'augmenter le chiffre d'affaires en attirant de nouveaux clients. Mais cette stratégie se révèle plus particulièrement efficace pour les entreprises qui sont déjà orientées clients et qui par conséquent connaissent bien les besoins et les exigences de ces derniers. Pour les autres, cette stratégie est plus susceptible d'augmenter les coûts sans améliorer les ventes et par conséquent de réduire la profitabilité.
La quatrième stratégie, assurer la disponibilité des produits, permet également d'accroître la rentabilité de l'entreprise en améliorant la fidélité des clients. Cependant, elle ne sera efficace que si les clients sont assurés de la fiabilité de l'entreprise. En effet, ces services ne valent pour les intermédiaires et les clients finaux que dans la mesure où ils permettent de garantir la disponibilité effective du produit et ce, en continu.

Dans la cinquième stratégie, la visée est d'offrir une expérience de consommation globale. A cause de la subjectivité des perceptions des consommateurs dans l'expérience qu'ils vivent lors de l'achat et de l'utilisation du produit, sa performance s'évalue surtout en termes de satisfaction. C'est pourquoi bien connaître les clients et leurs besoins et désirs est capital. Plus une entreprise sera orientée clients plus elle sera en mesure de les satisfaire et d'améliorer leur fidélité.

Offrir des services autour de ses produits, oui, mais pas dans n'importe quelles conditions, ni pour n'importe quel objectif. Ajouter indistinctement des services a de fortes chances d'augmenter les coûts pour l'entreprise sans en améliorer les ventes ni la rentabilité. Une telle stratégie ne sera efficace que si l'entreprise a clairement défini des objectifs de performance pour ces services, que ce soit en termes de rentabilité, de ventes ou de satisfaction des clients. De plus, il apparaît nécessaire que l'entreprise choisisse une offre de services qui corresponde aux caractéristiques de ses produits et de son environnement. 